\title{
CORRELATION BETWEEN THYROID DYSFUNCTION AND INFERTILITY
}

\author{
Lakshmi $G^{1}$, Assalatha $G^{2}$
}

${ }^{1}$ Associate Professor, Department of Physiology, Government Medical College, Trivandrum.

2Professor, Department of Physiology, Gokulam Medical College, Trivandrum.

\section{ABSTRACT}

\section{BACKGROUND}

Infertility affects approximately $15 \%$ of couples in the world. $35 \%$ of infertility are due to female causes and among female causes $59 \%$ contributed by ovarian dysfunction. There is a direct impact of thyroid hormone level on luteal function of the ovary. So, the thyroid function studies should be part of the evaluation of patients with persistent menstrual disorders. This inspired us to probe into this topic.

\section{METHODS}

In this prospective study, a group of fifty female patients with irregular periods attending the infertility clinic at Sree Avittom Thirunal Hospital selected as cases. Control group comprise fifty patients with regular periods of the same age group. The following parameters were studied - family history of thyroid dysfunction, body mass index, recent weight gain, serum thyroxine, triiodothyronine, Thyroid stimulating hormone, and Thyroid hormones estimated by radioimmunoassay. Statistical analysis was done using Pearson chi-square test.

\section{RESULTS}

Cases had positive family history of thyroid dysfunction (8\%), high body mass index (50\%), recent weight gain (42\%), 72\% cases are euthyroid with normal thyroid hormones levels. 28\% cases have clinical hyperthyroidism. Among $72 \%, 6 \%$ has subclinical hyperthyroid [Normal $\mathrm{T}_{3}$, $\mathrm{T}_{4}$, and low TSH. $(<0.6 \mu \mathrm{IU} / \mathrm{L})$ ] 8\% subclinical hypothyroid [Normal $\mathrm{T}_{3}$, $\mathrm{T}_{4}$, and high TSH $(>3.6 \mu \mathrm{IU} / \mathrm{L})$ ].

\section{CONCLUSION}

Estimation of serum TSH proves to be the most sensitive index of thyroid failure among other thyroid function tests. Hyperthyroidism or hypothyroidism whether clinical or subclinical has definite role in infertility. So, routine screening of TSH along with thyroid hormone is strongly recommended in the investigation for infertility.

\section{KEYWORDS}

Clinical Hyperthyroidism, Infertility, Subclinical Hyperthyroidism, Subclinical Hypothyroidism.

HOW TO CITE THIS ARTICLE: Lakshmi G, Assalatha G. Correlation between thyroid dysfunction and infertility. J. Evolution Med. Dent. Sci. 2016;5(65):4634-4638, DOI: 10.14260/jemds/2016/1056

\section{INTRODUCTION}

In a society increasingly conscious of individual rights and the quality of life, infertility is beginning to assume an importance approaching that of excess fertility.1,2 While steps are being taken to control the population growth on one side, there is a minor population of couples who seek treatment for infertility. ${ }^{3}$

There is a closely intertwined relationship between thyroid function and female reproductive axis. ${ }^{1,4} \mathrm{He}$ hypothesized that thyroid dysfunction influence both menstrual function and fertility through changes in sex hormone levels, gonadotropin release, and possibly ovarian functions. Higher TSH is also associated with increased incidence of abortion.1,5

Early changes of thyroid dysfunction could lead to subtle changes in ovulation, which then might have profound effects on infertility, but abnormal levels of thyroid hormones relate largely to changes in ovulation. ${ }^{6}$

Financial or Other, Competing Interest: None.

Submission 15-12-2015, Peer Review 11-03-2016,

Acceptance 17-03-2016, Published 12-08-2016.

Corresponding Author:

Dr. Lakshmi G,

Associate Professor,

Department of Physiology,

Government Medical College,

Trivandrum.

E-mail: lakshmi.syam92@gmail.com

DOI: $10.14260 /$ jemds/2016/1056
A physiological relationship exists between hypothalamic pituitary thyroid axis and hypothalamic pituitary ovarian axis and both act together as unified system.7,8 Maternal hyperthyroidism or hypothyroidism could affect the outcome of pregnancy producing a higher incidence of miscarriages, maternal complications, and thyroid disorders in women keeping in mind both menstrual irregularities and lactation failure. 9,10

Hence, the study was undertaken with the hope that incidence of infertility may be reduced by proper monitoring of the thyroid status. How hypothyroidism and hyperthyroidism affect the fertility of a woman? This is a question to be answered in the present scenario.

Hypothalamic pituitary thyroid axis comprises a group of physiologically interrelated endocrine organs that regulate and control the synthesis and secretion of thyroid hormones. The ultimate effector in this axis is the thyroid gland. ${ }^{11,12}$ TSH from the pituitary stimulate the secretion of both T4 and T3. These act at the pituitary level to control the secretion of TSH by a negative feedback mechanism. Secretion of TSH is stimulated by TRH from the hypothalamus. ${ }^{13}$

Hypothalamic pituitary ovarian axis comprises the GnRH secreted from arcuate nucleus of hypothalamus in a pulsatile pattern. The pulsatile secretion of GnRH from hypothalamus is responsible for maintaining a constant level of gonadotropins, FSH. ${ }^{14}$

There is a direct impact of thyroid regulation on luteal function of the ovary. He studied about the influence of thyroid 
hormones in the secretion of Gonadotrophic Releasing Hormone (GnRH).

TSH has a small FSH and LH like effect, which can cause ovulatory dysfunction. ${ }^{14}$ TSH possess a luteotropic activity and reach one fifth of the biological activity of human chorionic gonadotropin (hCG) ${ }^{15}$. Estimation of the serum TSH was proved to be most sensitive index of thyroid failure. Hypothalamus and pituitary might therefore be regarded as the most sensitive peripheral tissues in terms of reduced circulating thyroid.16,17

In a study on thyroid profile in infertile women, out of 47 women studied, 19.2\% women had hypothyroidism, $23.4 \%$ hyperthyroidism, and $57.4 \%$ euthyroid patients. Status of sex hormones was studied in hypothyroid subjects. $57 \%$ of them had high gonadotropin levels and hyperprolactinemia, high testosterone levels, which caused anovulation and 33\% had menorrhagia. Poor progesterone production associated with persistent endometrial proliferation might be responsible for massive bleeding. ${ }^{16,18}$ Another mechanism for this might be the failure of LH secretion. ${ }^{19,20}$

Infertility is defined as the inability to conceive after one year of regular intercourse without contraception. Infertility affects approximately $15 \%$ of couples in the world. According to a standard protocol, infertility evaluation usually identifies different causes including male infertility $(30 \%)$, female infertility (35\%), the combination of both $(20 \%)$, and finally unexplained or idiopathic infertility (15\%). Among the female causes of infertility, $59 \%$ is contributed by ovarian dysfunction. $21,22,23$

TSH assay because of its superior sensitivity and specificity was still preferred for screening subclinical thyroid disease associated with ovulatory dysfunction and infertility. ${ }^{23}$ Even slight hypothyroidism was associated with increased miscarriage, late fetal demise, and lower $1 \mathrm{Q}$ of offspring. ${ }^{24}$ Even slightly elevated TSH should be treated, though controversy remains. ${ }^{25}$ Treatment of hypothyroidism should be extended lifelong. ${ }^{26,27,28}$ There is the probable role of thyroid in a broad spectrum of reproductive disorders, abnormal sexual development, menstrual irregularities, infertility, etc. $29,30,31$

\section{MATERIALS AND METHODS}

\section{Selection of Patients}

Fifty female patients of reproductive age group with irregular periods attending the infertility clinic in Sree Avittom Thirunal Hospital were selected as study group. The control group comprise of fifty female patients reproductive age group with regular periods. Patients were selected based on inclusion criteria and exclusion criteria. Among those included were females of age between 20-40 years, not conceiving even after 1 year of unrestricted intercourse with irregular periods and no abnormality detected in their partners.

Those excluded were females of reproductive age group with regular periods, those with psychological problems, with congenital abnormalities of uterus, cervix, fallopian tube, those suffering from generalized illness, infections of cervix and uterus, fallopian tube and peritoneal cavity, endocrine disorders like hypothalamic dysfunction, pituitary failure, adrenal hyperplasia, androgen excess, diabetes. In females attending the infertility clinic, screening was done based on a proforma. Only known cases on treatment were included in the study.

\section{Parameters Studied}

The following parameters were studied. Family history of thyroid dysfunction, Body mass index, Recent weight gain, Thyroid enlargement, Hyperthyroidism, Hypothyroidism, Thyroid hormones.

\section{METHODS}

Immunoassay of thyroid hormones, T3, T4, TSH was done. T3 using RIAK-4/4A kit of BARC, T4 using 5/5A kit of BARC, TSH using immunoradiometric assay IRMARK-9.

\section{STATISTICAL ANALYSIS}

For the entry of the statistical data, the computer package used was Microsoft Excel. For analysis, SPSS of Windows Version 10 was used.

- P value of $<0.01$ was considered highly significant.

- P value of $<0.05$ was considered significant.

- P value of $>0.05$ was not considered to be statistically significant.

Association among variables were assessed using Pearson chi-square test.

All the parameters are statistically analysed and the tables are given below.

\section{RESULTS}

8\% among the cases show a positive family history of thyroid dysfunction and 92\% doesn't show a positive family history. (Table 1). Thus, the association of family history and thyroid dysfunction is found to be statistically significant.

$38 \%$ among the case group show values of body mass index greater than 25 whereas only $12 \%$ among the control group shows values greater than $25.80 \%$ of the control group and $50 \%$ of case group has body mass index with in the normal range. Thus, the association of infertility with body mass index is found to be statistically highly significant. (Table 2). $42 \%$ of cases show recent weight gain whereas none among the controls show recent weight gain. (Fig:1).

$72 \%$ cases have normal T3, T4 levels, $28 \%$ cases have raised $\mathrm{T} 3, \mathrm{~T} 4$ levels and none among the cases show decreased $\mathrm{T} 3$, T4. 86\% of the cases show TSH values with in the normal range, $8 \%$ with values greater than $3.6 \mathrm{IU} / \mathrm{L}$ and $6 \%$ with value less than $0.625 \mathrm{IU} / \mathrm{L}$. $76 \%$ among the control group show values within the normal range. Thus, there is statistically significant association between thyroid stimulating hormone and infertility. (Fig:3,4)

\section{DISCUSSION}

As regards the relationship of thyroid dysfunction and infertility, there is a strong positive correlation as evidenced by hyperthyroidism (28\%), euthyroidism (72\%), subclinical hyperthyroidism (6\%), and subclinical hypothyroidism (8\%).

Hypothalamus and pituitary maybe regarded as the most sensitive tissues in terms of reduced circulating thyroid hormone concentration. ${ }^{32}$ There are TSH as well as T3 receptors in ovary, which has an effect on steroidogenesis and oocyte maturation. ${ }^{33}$ Estimation of serum TSH proves to be the most sensitive index of thyroid failure. 34,35 TSH possess a luteotropic activity and reach one fifth of the biological activity of hCG. TRH or T3 or T4 do not possess luteotropic activity. 36,37

Subclinical hypothyroidism is found to be more prevalent in women compared to men. There is increased risk of developing overt hypothyroidism when TSH levels are greater 
than $12 \mathrm{mu} / \mathrm{L}$ associated with positive antithyroid antibodies. ${ }^{38,39}$ It is worth noting that TSH assay is warranted for all women planning pregnancy or those already pregnant. ${ }^{40,41}$

Even slightly elevated TSH should be treated as even slight hypothyroidism is associated with increased miscarriage, late foetal demise, and lower IQ of offspring. 42,43

In hypothyroidism, there is decreased T3, T4 levels, but increased TSH. Decreased T3, T4 levels cause a decrease in Sex Hormone-Binding Globulin (SHBG). A decrease in sex hormone-binding globulin not only cause a decrease in the bioactivity of bound hormones, but also an increase in the bioactivity of free hormones. But, the metabolic clearance rate of free estradiol is increased because of decreased binding to SHBG. This decreased free bioactive oestrogen result in lowering or disappearance of LH ovulatory peak, which result in lack of ovulation leading to ovarian dystrophy. 44

Hypothyroidism can also interfere with ovulation through an elevation in TRH. Low levels of thyroid hormones stimulate synthesis of TSH from anterior pituitary, which in turn cause increased secretion of TRH from hypothalamus. Elevated TRH can crosstalk with in pituitary gland to release other anterior pituitary hormone as prolactin. Elevated prolactin levels are known to interfere with ovulation either by decreased progesterone production from granulosa cells resulting in luteal phase defect or by an increase in dopamine (PRIF) levels by feedback inhibition. Increased dopamine can inhibit $\mathrm{GnRH}$ release. Decrease in GnRH release lead to a decrease in the secretion of FSH and LH leading to disruption of LH surge and anovulation. 45

Hyperthyroidism, increased levels of thyroid hormones lead to increased concentration of Sex Hormone-Binding Globulin (SHBG). As SHBG increases more and more oestrogen in the serum are bound to SHBG, so the level of free available estradiol in the serum decreases. Low levels of free oestrogen in the blood inhibit the release of GnRH by the hypothalamus and the secretion of LH and FSH by the anterior pituitary. So, there is no feedback of oestrogen on GnRH release, disruption of LH, and FSH surge leading to ovulatory dysfunction. ${ }^{46}$

Another mechanism as the cause for ovulatory dysfunction in hyperthyroid women is the decrease in metabolic clearance rate of estradiol, so the levels of free circulating oestrogens are increased. The conversion of androgens to oestrogens is increased. Finally, the oestrogen-androgen balance is modified with a higher unbound oestrogen/unbound testosterone ratio. Due to this oestrogen-androgen imbalance, there is supranormal setting of the hypothalamic gonadal axis. Hence, the hypothalamus may not respond to elevated levels of oestrogen as elevated levels of these hormones may be interpreted as normal by hypothalamus due to supranormal setting. LH surge will not occur leading to anovulation. ${ }^{47}$

Goitre or enlargement of thyroid gland can occur either in hypothyroidism and hyperthyroidism in infertile patients. An association is found between goitre and thyroid antibody status in infertile women. ${ }^{48}$ Goitre subsided with initiation of iodine and/or L-thyroxine therapy. But, in subclinical hypothyroidism, there is no increase in thyroid volume and iodine avidity is also decreased. In the present study, a supportive correlation exists between goitre and infertility as evidenced by $22 \%$ cases with thyroid enlargement and $78 \%$ cases with no thyroid enlargement.(Fig.2) ${ }^{48}$
The observation in the present study points to the fact that a closely intertwined relationship exists between thyroid function and the female reproductive axis. Thyroid dysfunction influence both menstrual function and fertility.

Similarly, alterations in reproductive physiology can also modulate thyroid function.

The chance of infertility is more among women with combined thyroid dysfunction and infertility when compared to other causes of infertility. The chance of conception is further decreased in the presence of antithyroid antibodies. Treatment of these infertile women with pure thyroid hormones lead to the improvement of menstrual cycles and also to desired conception. Patient should be continuously monitored even after conception because maternal hyperthyroidism or hypothyroidism can affect the outcome of pregnancy producing a higher incidence of miscarriages, maternal complications, and congenital malformations. Fetal/neonatal hypothyroidism or hyperthyroidism produced by the transplacental passage of maternal thyroid auto antibodies can impair growth and neuropsychological development of affected children. ${ }^{48,49}$

Ideally, complete follow up of all the patients attending the infertility clinic should be done, which is not possible during the short span of the present study. Hope, this study is successful in the explanation of thyroid-ovary relation and add to the practical clinical application of experimental knowledge of human reproduction. It is our hope that the future workers in this field would be benefited with more insights into the enigma that thyroid dysfunction continues to alleviate the anguish of the couples concerned and those in the medical profession alike.

This study supports the possible role of hyperthyroidism and hypothyroidism in infertility. This is probably due to change in the levels of SHBG, which brings about the change in the free oestrogen level in the serum. Altered oestrogen levels can change the serum FSH and LH levels. This in turn can lead to ovulatory dysfunction and infertility. ${ }^{50}$

So, appropriate screening of all infertile patients is recommended routinely to evaluate pituitary function by thyroid function studies. On treatment with thyroxine, there is better chance of conception. The screening and treatment of these patients should be continued even after conception. These might help in the early initiation of corrective measures that prevent or limit damage to mother and foetus.

\section{CONCLUSION}

So, to conclude, thyroid dysfunction either hyperthyroidism or hypothyroidism has a definite role in infertility and hypothalamic pituitary thyroid axis play a vital role in gonadal function. Hence, it is recommended to have a routine screening for thyroid dysfunction for all cases of infertility. Although, there have been tremendous advances in the treatment of infertility. It is a matter of frustration for all concerned that a successful outcome cannot be guaranteed.

It is in this context, the study becomes significant. Screening for thyroid function test should be advocated in all infertile clinics. Abnormality of any can be treated at the earliest and the treatment is miraculously successful. This study may throw light for all the couples concerned who seek treatment for infertility. 


\section{Limitations of the Study}

The study is of short duration. Due to unavoidable circumstances, we were not able to do further follow up of these patients.

\section{ACKNOWLEDGEMENT}

I express my sincere thanks and deep gratitude to Dr. Assalatha. G, Professor and Head of the Department of Physiology who despite her own workload found time in guiding me in my research work and for being committed as my guide. I am greatly indebted to her for her constructive criticism, expert guidance, and encouragement in carrying out this work.

Words cannot fully express my gratitude and thanks to late Dr. Sumaprabha. K.S, Associate Professor, and my co-guide for her expert guidance with unfading and sustained encouragement and support during the various stages of the present work.

I would like to thank Dr. Sheela Balakrishnan, Assistant Professor of Obstetrics and Gynaecology, SAT Hospital who found time off her busy schedule for giving invaluable guidance at different stages of my research work.

I sincerely thank the lab technicians of the Department of Nuclear Medicine for helping me to conduct the tests.

I am immensely thankful to Dr. Kurian Mathew for helping me with the statistical analysis.

This work wouldn't have been materialized or the cooperation of the patients attending the infertility clinic in spite of their mental agony and social stigma. My heartfelt thanks, one and all.

\begin{tabular}{|c|c|c|}
\hline \multirow{2}{*}{$\begin{array}{c}\text { Family } \\
\text { History }\end{array}$} & Gases & Control \\
\cline { 2 - 3 } & $4692.0 \%$ & $50100 \%$ \\
\hline No & $48.0 \%$ & 0 \\
\hline Yes & \multicolumn{2}{|c|}{ Table 1: Family History } \\
\hline
\end{tabular}

Chi-square test, Value-P value, with Yates correction 4.167, 0.041 Significant

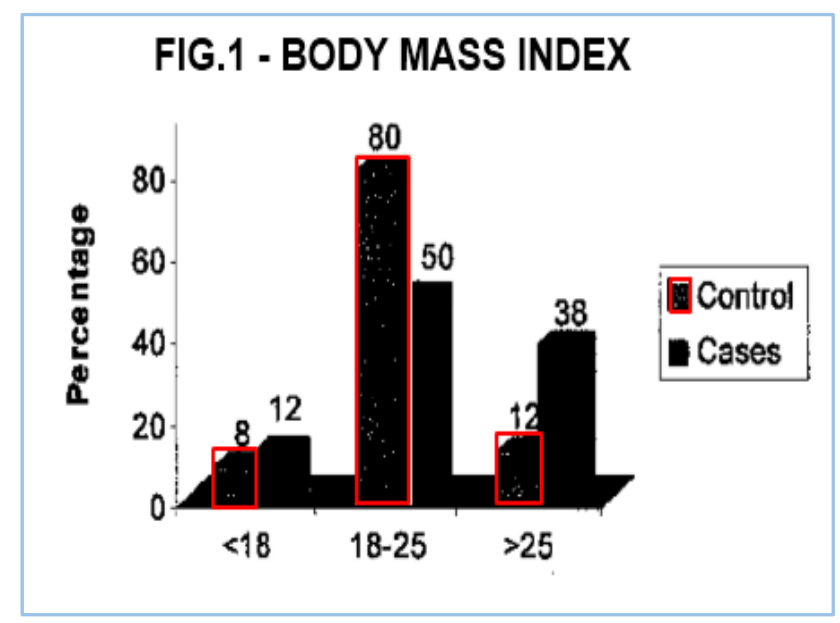

Fig. 1: Body Mass Index

\begin{tabular}{|c|c|c|}
\hline Thyroid & \multicolumn{2}{|c|}{ Rrnnn } \\
\hline & Cases & Control \\
\hline Not Enlarged & $3978.0 \%$ & $50100 \%$ \\
\hline Enlarged & $1122.0 \%$ & 0 \\
\hline \multicolumn{2}{|c|}{ Table 2: Thyroid Enlargement } \\
\hline
\end{tabular}

Chi square test Value, P. Value, Very highly, 12.36, 0.000, Significant

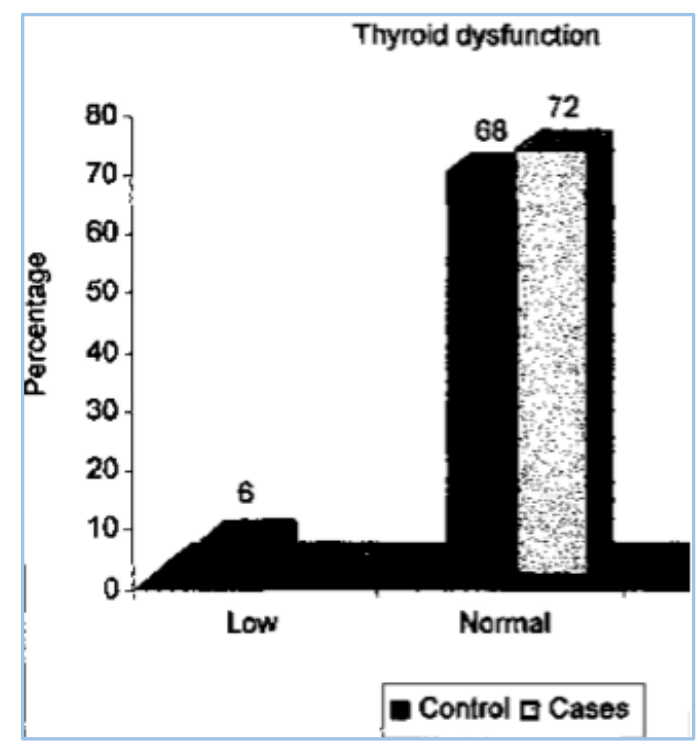

Fig. 2

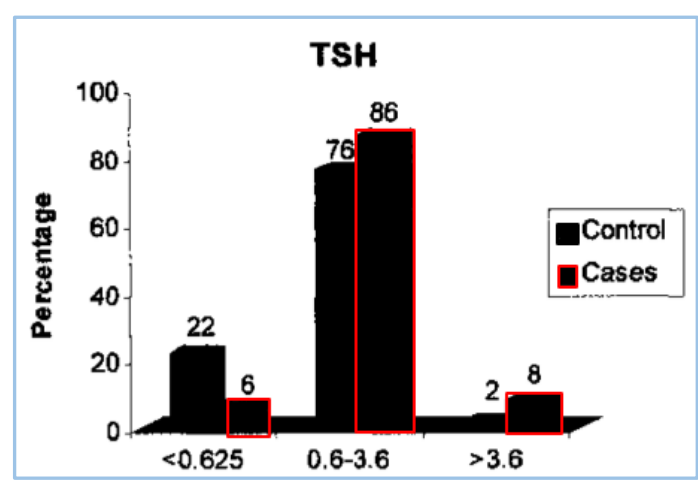

Fig. 3

\section{REFERENCES}

1. Adlersberg MA, Burrow GN. Focus of primary care. Thyroid function and dysfunction in women. Obstet Gynaecol Surv 2002;57(3):S1-7.

2. Arojoki M, Jokimaa $V$, Juuti A, et al. Hypothyroidism among infertile women in Finland. Gynaecol Endocrinol 2000;14(2):127-31.

3. Baird DT. Endocrinology of female infertility. British Medical Bulletin 1979;35(2):193-8.

4. Baird DT, Fraser IS. Disorders of the hypothalamic pituitary ovarian axis. Clin Endocrinol Metab 1973;2(3):469-88.

5. Bartaleva L, Martino E, Falcone M, et al. Evaluation of the nocturnal serum thyrotropin (TSH) surge, as assessed by TSH ultrasensitive assay in patients recovering long-term L-thyroxine suppressive therapy and in patients with various thyroid disorders. Clin Endocrinol Metab 1987;65(6):1265-71.

6. Carnetti N, Mincei D, Casoli M Attual. Relations between ovarian and thyroid function, oestrogenism and use of $\mathrm{I}^{131}$ Obstet Gynaecol 1968;1:155-64.

7. Carnetti N, Predin G. Relations between thyroid and ovarian function oestrogenism and basal metabolism. Attual Obstet Gynaecol 1968;1:111-20. 
8. Chiovato L, Lapi P, Fiore E, et al. Thyroid autoimmunity and female gender. Journal of Endocrinological investigation 1993;16(5):384-91.

9. Doufgas AG, Mastorakos G. The hypothalamic pituitary thyroid axis and the female reproductive system. Ann N Y Acad Sci 2000;900:65-75.

10. Chiovato L, Tonacchera M, Lapi P, et al. Thyroid autoimmunity and neuropsychological development. Acta Med Austriaca 1992;19 Suppl 1:91-5.

11. Edwards CR, Forsyth SA, Bosmer GM. Amenorrhea, galactorrhea and primary hypothyroidism with high circulating levels of prolactin. British Medical Journal 1971;3:462-4.

12. Evered DC, Ormston BJ, Smith PA, et al. Grades of hypothyroidism. British Medical Journal 1973;1(5854):657-62.

13. Evers JL. Female infertility. Lancet 2002;360(9327):151-9.

14. Forti G, Karusz C. Evaluation and treatment of the infertile couple. Clin Endocrinol Metab 1998;83(12):4177-88.

15. Georgopoulos NA, Markon KB, Pappas AP. Ovulation induction with pulsatile gonadotropin releasing hormone (GnRH) or gonadotropins in a case of hypothalamic amenorrhoea and diabetic insipidus. Gynaecol Endocrinol 2001;15(6):421-5.

16. Gerhard I, Becker T, Eggert-Kruse W, et al. Thyroid and ovarian function in infertile women. Human Reproduction 1991;6(3):338-45.

17. Glass AR, Dahms WT, Abraham G, et al. Secondary amenorrhoea in obesity: etiological role of weight related androgen excess. Fertil steril 1978;30(2):243-4.

18. Glass AR. Endocrine aspects of obesity. Med Clin North Am 1989;73(1):139-60.

19. Grassi G, Balsamo A, Ansaldi C, et al. Thyroid autoimmunity and infertility. Gynaecol Endocrinol 2001;15(5):389-96.

20. Gruters A, Krude $\mathrm{H}$, Biefermann $\mathrm{H}$, et al. Alterations of neonatal thyroid function. Acta Paediatr Suppl 1999;88(428):17-22.

21. Isaksson R, Tiitinen A. Present concept of unexplained infertility. Gynaecol Endocrinol 2004;18(5):278-90.

22. Johnson CA. Thyroid issues in reproduction. Clin Tech small Anim Pract 2002;17(3):129-32.

23. Kalro BN. Impaired fertility caused by endocrine dysfunction in women. Endocrinol Metab Clini North Am 2003;32(3):573-92.

24. Kopelman PG, Pilkington TRE, White N, et al. Abnormal sex steroid secretion and binding in massively obese women. Clin Endocrinol 1980;12(4):363-9.

25. Koutras DA. Disturbance of menstruation in thyroid disease. Ann N Y Academic Science 1997;816:280-4.

26. Krassas GE. Thyroid disease and female reproduction. Fertil Steril 2000;74(6):1063-70.

27. Lakshmi Singh CG, Agarwal SR, Chowdary P. Thyroid profile in infertile women. Ind Journal of Obstetrics and Gynaecology 1990;37(12):248-53.

28. Lincoln SR, Ke RW, Kutteh WH. Screening for hypothyroidism in infertile women. Journal of Reproductive Medicine 1999;44(5):455-7.

29. Louvet JP, Gouarre M, Salandini AM, et al. Hypothyroidism and anovulation. Lancet 1979;12(8124):1032.
30. McClure RD. Endocrine investigation and therapy. Uro Clin North Am 1987;14(3):471-88.

31. Moran C, Huerta R, Azziz R. Infetility treatment before assisted reproductive techniques. Gynaecol obstet Mex 2001;69:167-71.

32. Newmark SR, Rossinii AA, Naftolin F, et al. Gonadotropin profiles in fed and fasted obese women. Am J Obstet Gynaecol 1979;133(1):75-80.

33. Oravec S, Hlavacka S. Disorders of thyroid function and fertility disorders. Cecka Gynaecol 2000;65(1):53-7.

34. Pharoah PO, Elles SM, Ekins RP, et al. Maternal thyroid function, iodine deficiency and fetal development. Clin Endocrinol (oxf) 1976;5(2):159-66.

35. Poppe K, Glinoer D. Thyroid autoimmunity and hypothyroidism before and during pregnancy. Human reproduction update 2003;9(2):149-61.

36. Poppe K, Glionoor D, Van Steirleghem A, et al. Thyroid dysfunction and autoimmunity in infertile women. Thyroid 2002;12(11):997-1001.

37. Poppe K, Velkaniers B. Female infertility and the thyroid. Best Pract Res Clin Endocrinol Metab 2004;18(2):153-65.

38. Poppe K, Velkeniors BV. Thyroid disorder in infertile women. Ann Endocrinol (Paris) 2003;64(1):45-50.

39. Raber W, Gessl A, Nowotny P, et al. Hyperprolactinemia in hypothyroidism: clinical significance and impact of TSH normalization. Endocrinol (Oxf) 2003;58(2):185-91.

40. Raber W, Nowotny P, Vytiska-Binstorfer E, et al. Thyroxine treatment modified in infertile women according to thyroxine releasing hormone testing: 5 year follow up of 283 women referred after exclusion of absolute causes of infertility. Human reproduction 2003;18(4):707-14.

41. Redmond GP. Thyroid dysfunction and women's reproductive health. Thyroid 2004;14(1):S5-15.

42. Sato T, Miyagawa K, Igarashi N, et al. Alternating hyper and hypothyroidism with thyroid stimulating and TSH-binding inhibition immune globulins. Acta Paediatr Jpn 1987;29(6):862.

43. Sesnova EA. The role of thyroid in female reproductive system. Akush Ginekol (Mosk) 1989;4:6-11.

44. Shalev E, Eliyahu S, Ziv M, et al. Routine thyroid function tests in infertile women, are they necessary. Horm Metab Res 2001;33(4):216-20.

45. Shalev E, Eliahun S, Zev M, et al. Routine thyroid function testing in infertile women? are they necessary? American Journal of Obstetrics and Gynaecology 1994;171(5): 1191-2.

46. Speroff L. The effect of aging on fertility. Curr Opin Obstet Gynaecol 1994;6(2):115-20.

47. Stratford GA, Barth JH, Rutherford AJ, et al. Value of thyroid function tests in routine screening of women investigated for infertility. Human Fertil (Camb) 2000;3(3):203-6.

48. Stratford GA. Physiopathological determinants of human infertility. Human Reproduction update 2002;8(5):435-47.

49. Thomas R, Ried R. Thyroid disease and reproductive dysfunction, a review. Obstet Gynaecol 1987;70(5):78998.

50. Vaidya R, Shringi M. Thyroid and female reproduction. Journal of Postgraduate Medicine 1993;39(3):118-9. 